\title{
Differential telomerase expression in idiopathic pulmonary fibrosis and non-small cell lung cancer
}

\author{
K.M. ANTONIOU ${ }^{1,2}$, K.D. SAMARA ${ }^{1,2}$, I. LASITHIOTAKI ${ }^{2}$, G.A. MARGARITOPOULOS ${ }^{1,2}$, G. SOUFLA ${ }^{3}$, \\ I. LAMBIRI ${ }^{1}$, I. GIANNARAKIS ${ }^{1}$, I. DROSITIS ${ }^{4}$, D.A. SPANDIDOS ${ }^{3}$ and N.M. SIAFAKAS ${ }^{1}$ \\ ${ }^{1}$ Department of Thoracic Medicine, ${ }^{2}$ Laboratory of Molecular and Cellular Pulmonology, ${ }^{3}$ Laboratory of Clinical Virology, \\ and ${ }^{4}$ Department of Thoracic Surgery, Medical School, University of Crete, 71110 Heraklion, Crete, Greece
}

Received August 1, 2013; Accepted September 20, 2013

DOI: $10.3892 /$ or.2013.2753

\begin{abstract}
Telomerase is a reverse transcriptase ribonucleoprotein (h-TERT) that synthesizes telomeric repeats using its RNA component (h-TERC) as a template. Telomerase dysfunction has been associated with both fibrogenesis and carcinogenesis. In this study, we aimed to evaluate the telomerase mRNA expression levels of both subunits (h-TERT and h-TERC) in lung tissue and bronchoalveolar lavage fluid (BALF) from patients with idiopathic pulmonary fibrosis (IPF) and nonsmall cell lung cancer (NSCLC), since there are indications of common pathogenetic pathways in these diseases. We prospectively examined lung tissue samples from 29 patients with IPF, 10 patients with NSCLC and 21 controls. Furthermore, we examined BALF samples from 31 patients with NSCLC, 23 patients with IPF and 12 control subjects. The mRNA expression for both $\mathrm{h}$-TERT and h-TERC was measured by real-time RT-PCR. In the lung tissue samples, both h-TERT and h-TERC mRNA expression levels varied among the 3 groups $(p=0.036$ and $p=0.002$, respectively). h-TERT mRNA levels in the patients with IPF were lower compared with those in the controls $(p=0.009)$ and patients with NSCLC ( $\mathrm{p}=0.004)$. h-TERC mRNA levels in the patients with IPF were lower compared with those in the controls $(\mathrm{p}=0.0005)$ and patients with NSCLC $(\mathrm{p}=0.0004)$. In the BALF samples, h-TERT mRNA expression levels varied among the groups $(\mathrm{p}=0.012)$. More specifically, h-TERT mRNA levels in the patients with IPF were higher compared with those in the controls $(p=0.03)$ and patients with NSCLC $(p=0.007)$. The attenuation of telomerase gene expression in IPF in comparison to lung cancer suggests a differential role of this regulatory gene in fibrogenesis and carcinogenesis. Further functional studies are required in order to further elucidate the role of telomerase in these devastating diseases.
\end{abstract}

Correspondence to: Dr K.M. Antoniou, Department of Thoracic Medicine, University Hospital, Medical School, University of Crete, 71110 Heraklion, Crete, Greece

E-mail: kantoniou@med.uoc.gr

Key words: telomerase, fibrogenesis, carcinogenesis, bronchoalveolar lavage fluid, lung tissue

\section{Introduction}

Idiopathic pulmonary fibrosis (IPF), accounting for $>50 \%$ of cases with idiopathic interstitial pneumonia (IIP), is a progressive, lethal disease, whose etiology remains enigmatic $(1,2)$. The majority of IPF cases are sporadic, while 2-20\% of cases are familial, inherited in an autosomal dominant pattern with incomplete penetration (2). The current hypothesis supports the notion that IPF is not an inflammatory disorder, but a complex process characterized by abnormal pneumocyte apoptosis and the profound disruption of the renewal of the alveolar epithelium, making it, at least in many aspects, quite similar to malignant lung disease (2-4).

IPF and lung cancer have several striking similarities. Both are fatal lung diseases, whose main event is aberrant cell proliferation and they share a number of pathogenetic pathways (4-6). Genetic alterations, response to growth and inhibitory signals, resistance to apoptosis, myofibroblast origin and behavior, altered cellular communication and intracellular signaling pathways are all fundamental pathogenic hallmarks of both IPF and cancer (4-6). Furthermore, both diseases are characterized by the lack of effective treatment and a poor survival rate, a combination that underlines the need for further research for novel information and perspectives (2,4-6).

Mutations of various genes have emerged in search of the etiology of both diseases, such as the 'aging' gene, telomerase $(3,4)$. Telomeres are repetitive DNA sequences at the end of chromosomes, which protect them from degradation, irregular recombination and end-to-end fusions (3). Telomeres decrease in length with every cell division until they reach a critically short size and signal the arrest of cell proliferation, senescence and apoptosis (5). The active telomerase ribonucleoprotein complex contains 3 subunits: the telomerase reverse transcriptase (h-TERT), the RNA subunit (h-TERC) and dyskerin (6).

The catalytic activity of this enzyme resides in the h-TERT component, and thus the regulation of h-TERT mRNA expression seems to be the most important step for telomerase activation (7). Although h-TERT seems to be the key component in telomerase regulation and telomere synthesis, h-TERC is also required to maintain cell growth, particularly when h-TERT is overexpressed (8). It appears that h-TERC may play a stabilizing role in the telomerase complex. h-TERT is highly 
expressed in germ cells, cells with proliferative potential and in immortalized cancer cells (9-11), although in most other cells, telomerase activity is restricted in humans (12). In addition, in IPF, $25 \%$ of individuals who have either familial or sporadic pulmonary fibrosis, without h-TERT or h-TERC mutation, have shorter telomeres in their circulating leukocytes (2).

Since there are indications of common pathogenetic pathways between IPF and lung cancer, in this study, we aimed to evaluate the expression levels of both telomerase subunits (h-TERT and h-TERC) in lung tissue and bronchoalveolar lavage fluid (BALF) from patients with IPF and non-small cell lung cancer (NSCLC).

\section{Patients and methods}

Patients. Consecutive patients with IPF and NSCLC from the Department of Thoracic Medicine, University Hospital of Heraklion, Crete, Greece were enrolled in this study. The diagnosis for IPF was based on internationally accepted clinical and imaging criteria, using video-assisted thoracoscopic surgery (VATS), where needed (13). All patients were sporadic IPF cases. The diagnosis for NSCLC was based on histopathological criteria from bronchial biopsies and cytology from bronchial washings. The patients included in this study were classified as NSCLC according to the WHO criteria (1997). All IPF and NSCLC patients were newly diagnosed and treatment naive at the time of either bronchoscopy or surgery.

The lung tissue samples were obtained from 29 patients with IPF, 10 patients with NSCLC and 21 controls. NSCLC samples were obtained from sections of the lung with verified positive histology. Control lung tissue specimens were obtained from patients undergoing lobectomy or pneumonectomy for bronchogenic carcinoma, at a macroscopically healthy site distant from the malignancy. The samples were further verified histologically as free of malignancy before being classified as control samples, as previously described by ours and other groups $(14,15)$. The BALF samples were obtained from 23 patients with IPF, 31 patients with NSCLC and 12 controls. Control subjects were patients undergoing bronchoscopy for the investigation of haemoptysis, without any pulmonary comorbidities and with normal bronchoscopic findings and cytology results. Subjects who had experienced respiratory infections during the 6 weeks prior to bronchoscopy or surgery were excluded from this study. All patients were of comparable age. The patients with NSCLC and the control subjects exhibited near normal pulmonary function tests, whereas the patients with IPF had a mild restriction with decreased pulmonary volumes. Demographics and pulmonary function tests of the patients and controls are presented in Table I.

Ethics statement. Informed consent was obtained from all patients and control subjects who participated in this study. The study protocol was approved by the Ethics Committee of the University Hospital of Heraklion.

Biological samples and processing. BALF was obtained from all patients at room temperature as previously described (16). After filtering through a sterile gauze (Thompson, Ontario, Canada) to remove debris, BALF from each patient was centrifuged at $400 \mathrm{x} \mathrm{g}$ for $15 \mathrm{~min}$ at $4^{\circ} \mathrm{C}$ and the supernatant and pellet were stored at $-80^{\circ} \mathrm{C}$. Lung tissue biopsy specimens were obtained at room temperature, immediately frozen in liquid nitrogen and stored at $-80^{\circ} \mathrm{C}$.

Gene expression. BALF pellets and homogenized lung tissue samples were processed using the TRIzol reagent (Invitrogen, Carlsbad,CA, USA) protocol for total RNA extraction according to the manufacturer's instructions. RNA concentration and purity were evaluated using a spectrophotometer. Aliquots of RNA were stored at $-80^{\circ} \mathrm{C}$ until use. cDNA from each sample was derived by reverse transcription of $2 \mu \mathrm{g}$ of total RNA using the AffinityScript ${ }^{\mathrm{TM}}$ Multi Temperature cDNA synthesis kit, (Stratagene, La Jolla, CA, USA). Random hexamers were used as amplification primers. To remove the RNA template, cDNA was incubated with $E$. coli $\mathrm{RNaseH}$ and stored at $-20^{\circ} \mathrm{C}$ until use.

Transcript levels of h-TERT, h-TERC and transforming growth factor (TGF)- $\beta$ were determined using the Mx3000P Real-Time PCR system (Stratagene) and SYBR-Green I Master Mix (Stratagene) according to the manufacturer's instructions, as previously described (17-19). All primers were designed to span at least one intron in order to avoid amplification of contaminating genomic DNA. $\beta$-globin was used as an internal control to normalize mRNA expression levels, as previously described by our study group (17-19). To verify the results of the melt curve analysis, PCR products were analyzed by electrophoresis on $2 \%$ agarose gels stained with ethidium bromide and photographed on a UV light transilluminator. Primer sequences and annealing temperatures for all the genes analyzed, as well as for $\beta$-globin are presented in Table II.

Statistical analysis. The one sample Kolmogorov-Smirnov test was employed to assess normality. Data were compared using Kruskal-Wallis ANOVA with the Mann-Whitney test for post-hoc comparisons. The percentages of patients expressing hTERT, h-TERC and TGF- $\beta$ were compared using the $\chi^{2}$ test. Values are expressed as the median (lower-upper quartiles) and a value of $\mathrm{p}<0.05$ was considered to indicate a statistically significant difference. Linear regression analysis (Spearman's rank correlation coefficient) was used to assess TGF- $\beta$ expression in the lung tissue. Statistical calculations were performed using Statistica 7 software (StatSoft, Tulsa, OK, USA).

\section{Results}

Gene expression in lung tissue. As regards the h-TERT subunit, the telomerase gene was expressed in $52.4 \%$ of the controls, $13.8 \%$ of the patients with IPF and $60 \%$ of the NSCLC population (Table III). As regards the h-TERC subunit, the telomerase gene was expressed in a significantly lower number of patients with IPF compared with the controls and patients with NSCLC (Table III).

h-TERT mRNA expression levels differed among the 3 groups $(p=0.036)$. h-TERT mRNA levels in the patients with IPF were significantly lower compared with those in the controls $(\mathrm{p}=0.009)$ and patients with NSCLC $(\mathrm{p}=0.004)$. The majority of subjects in all 3 groups expressed h-TERC mRNA (Table IVA). h-TERC mRNA expression levels differed among the 3 groups $(\mathrm{p}=0.002)$. Again, h-TERC mRNA levels in the patients with IPF were significantly lower compared with those in the controls 
Table I. Clinical characteristics and pulmonary function tests of all patients studied.

A, Lung tissue samples

\begin{tabular}{|c|c|c|c|c|}
\hline Characteristics & Controls & IPF & NSCLC & p-value \\
\hline No. & 21 & 29 & 10 & \\
\hline $\mathrm{Age}^{\mathrm{a}}$ & $63.41 \pm 4.12$ & $67.8 \pm 3.93$ & $59.38 \pm 6.81$ & $\mathrm{p} 1, \mathrm{p} 2, \mathrm{p} 3=\mathrm{NS}$ \\
\hline Gender (M/F) & $17 / 4$ & $21 / 8$ & $9 / 1$ & \\
\hline Non-smoker & 9 & 13 & 0 & \\
\hline Smokers & 6 & 5 & 8 & \\
\hline Ex-smokers & 6 & 11 & 2 & \\
\hline $\mathrm{FEV}^{\mathrm{a}}$ & $85.31 \pm 8.49$ & $75.64 \pm 4.23$ & $83.78 \pm 5.74$ & $\mathrm{p} 1, \mathrm{p} 2, \mathrm{p} 3=\mathrm{NS}$ \\
\hline $\mathrm{FVC}^{\mathrm{a}}$ & $93.22 \pm 7.50$ & $74.85 \pm 3.30$ & $92.19 \pm 8.25$ & $\mathrm{p} 1<0.05, \mathrm{p} 2, \mathrm{p} 3=\mathrm{NS}$ \\
\hline $\mathrm{FEV} / \mathrm{FVC}^{\mathrm{a}}$ & $73.33 \pm 4.29$ & $82.14 \pm 1.85$ & $71.89 \pm 4.91$ & $\mathrm{p} 1, \mathrm{p} 3<0.05, \mathrm{p} 2=\mathrm{NS}$ \\
\hline $\mathrm{DLCO}^{\mathrm{a}}$ & $74.43 \pm 11.37$ & $50.73 \pm 4.30$ & - & $\mathrm{p} 1<0.05$ \\
\hline
\end{tabular}

B, BALF samples

\begin{tabular}{lcccc}
\hline Characteristics & Controls & IPF & NSCLC & p-value \\
\hline No. & 12 & 23 & 31 & $\mathrm{p} 1, \mathrm{p} 2, \mathrm{p} 3=\mathrm{NS}$ \\
Age $^{\mathrm{a}}$ & $61.75 \pm 3.51$ & $69.17 \pm 1.23$ & $66.62 \pm 1.74$ & \\
Gender (M/F) & $10 / 2$ & $18 / 5$ & $27 / 4$ & \\
Non-smoker & 5 & 9 & 18 & $\mathrm{p} 1, \mathrm{p} 2, \mathrm{p} 3=\mathrm{NS}$ \\
Smokers & 4 & 3 & 11 & $\mathrm{p} 1, \mathrm{p} 3<0.05, \mathrm{p} 2=\mathrm{NS}$ \\
Ex-smokers $_{\text {FEV1 }}^{\text {a }}$ & 3 & 11 & $92.34 \pm 4.71$ & $\mathrm{p} 1, \mathrm{p} 3<0.05, \mathrm{p} 2=\mathrm{NS}$ \\
FVC $^{\text {a }}$ & $86.78 \pm 8.71$ & $77.88 \pm 3.45$ & $72.14 \pm 5.23$ & $\mathrm{p} 1<0.05$ \\
FEV1/FVC $^{\text {a }}$ & $91.88 \pm 6.90$ & $71.68 \pm 4.52$ & - & \\
DLCO $^{\text {a }}$ & $75.41 \pm 5.19$ & $85.31 \pm 1.79$ & $52.69 \pm 4.13$ & \\
\hline
\end{tabular}

Values are expressed as the means \pm SEM (standard error of the mean). ${ }^{a}$-test; $p<0.05$ was considered to indicate a statistically significant difference. NS, not significant. p1, IPF vs. controls; p2, NSCLC vs. controls; p3, IPF vs. NSCLC. NSCLC, non-small cell lung cancer; IFP, idiopathic pulmonary fibrosis; BALF, bronchoalveolar lavage fluid; M, male; F, female; FEV1, forced expiratory volume in 1 sec; FVC, forced vital capacity; DLCO, diffusing capacity for carbon monoxide.

Table II. Primer sequences used for real-time RT-PCR.

\begin{tabular}{lllc}
\hline Gene & & Primer pair sequence $\left(5^{\prime} \rightarrow 3^{\prime}\right)$ & Annealing temperature $\left({ }^{\circ} \mathrm{C}\right)$ \\
\hline TGF- $\beta$ & $\begin{array}{l}\text { Forward } \\
\text { Reverse }\end{array}$ & AAGGACCTCGGCTGGAAGTGC & 62 \\
h-TERT & Forward & TGACACCTCACCTCACCCAC \\
& Reverse & CACTGTCTTCCGCAAGTTCAC & 51 \\
h-TERC & Forward & GCCTGCCGCCTTCCACCGTTCATT & 59 \\
$\beta$-globin & Reverse & GACTCGCTCCGTTCCTCTTCCTG & 58 \\
& Forward & GCTTCTGACACAACTGTGTTCACTAGC & 58 CACCAACTTCATCCACGTTCACC \\
\hline
\end{tabular}

$(\mathrm{p}=0.0005)$ and patients with NSCLC $(\mathrm{p}=0.0004)$ (Table IVA, Fig. 1).

TGF- $\beta$ mRNA expression levels differed among the 3 groups $(p<0.0001)$. TGF- $\beta$ mRNA levels in the patients with IPF were significantly higher compared with those in the controls and patients with NSCLC (Table IVA). Using linear regression analysis (Spearman's rank correlation coefficient), we assessed that TGF- $\beta$ expression in the lung tissue positively 
Table III. Percentage of subjects expressing h-TERT and h-TERC mRNA in lung tissue and BALF samples.

A, Lung tissue samples

\begin{tabular}{ccccl}
\hline & $\begin{array}{c}\text { Control } \\
(\mathrm{n}=21)\end{array}$ & $\begin{array}{c}\text { IPF } \\
(\mathrm{n}=29)\end{array}$ & $\begin{array}{c}\text { NSCLC } \\
(\mathrm{n}=10)\end{array}$ & p-value \\
\hline h-TERT & $52.4 \%$ & $13.8 \%$ & $60 \%$ & $\begin{array}{l}\mathrm{p} 1=0.003 \\
\mathrm{p} 2=\mathrm{NS} \\
\mathrm{p} 3=0.004\end{array}$ \\
& & & & \\
h-TERC & $61.9 \%$ & $17.3 \%$ & $90 \%$ & $\begin{array}{l}\mathrm{p} 1=0.001 \\
\mathrm{p} 2=\mathrm{NS} \\
\mathrm{p} 3=0.0001\end{array}$ \\
& & & & \\
\hline
\end{tabular}

B, BALF samples

\begin{tabular}{lcccl}
\hline & $\begin{array}{c}\text { Control } \\
(\mathrm{n}=12)\end{array}$ & $\begin{array}{c}\text { IPF } \\
(\mathrm{n}=23)\end{array}$ & $\begin{array}{c}\text { NSCLC } \\
(\mathrm{n}=31)\end{array}$ & $\mathrm{p}$-value \\
\hline h-TERT & $33 \%$ & $65.2 \%$ & $25.8 \%$ & $\begin{array}{l}\mathrm{p} 1=0.07 \\
\mathrm{p} 2=\mathrm{NS} \\
\mathrm{p} 3=0.004\end{array}$ \\
& & & & $\mathrm{p} 1, \mathrm{p} 2, \mathrm{p} 3=\mathrm{NS}$
\end{tabular}

${ }^{\mathrm{a}} \chi^{2}$ test, $\mathrm{p}<0.05$ was considered to indicate a statistically significant difference. NS, not significant p1, IPF vs. controls; p2, NSCLC vs. controls; p3, IPF vs. NSCLC. NSCLC, non-small cell lung cancer; IFP, idiopathic pulmonary fibrosis; BALF, bronchoalveolar lavage fluid.

correlated with $\mathrm{h}$-TERT and $\mathrm{h}$-TERC expression in a very small percentage of patients with IPF and NSCLC (Table V).

Gene expression in BALF. The percentage of the patients with NSCLC and control subjects expressing h-TERT was lower than that of patients with IPF $(\mathrm{p}<0.05$, Table III). The percentages of h-TERC mRNA expression did not differ significantly among the groups (Table III).

h-TERT mRNA expression levels varied among the groups $(\mathrm{p}=0.012)$ (Table IVB). More specifically, h-TERT mRNA levels in the patients with IPF were significantly higher compared with those in the controls $(p=0.03)$ and patients with NSCLC ( $p=0.007)$. h-TERC mRNA expression levels varied among the 3 groups $(\mathrm{p}=0.07)$. Post-hoc analysis revealed that $\mathrm{h}$-TERC mRNA levels in the patients with IPF tended to be higher compared with those in the control subjects $(\mathrm{p}=0.08)$ (Table IVB, Fig. 2).

TGF- $\beta$ mRNA expression levels differed among the 3 groups $(\mathrm{p}<0.0001)$ (Table IVB). Using linear regression analysis (Spearman's rank correlation coefficient), we assessed that TGF- $\beta$ expression in the BALF samples negatively correlated with $\mathrm{h}$-TERT and $\mathrm{h}$-TERC expression in a very small percentage of patients with IPF (Table V). On the contrary, TGF- $\beta$ expression positively correlated with h-TERC in the BALF samples from patients with NSCLC with a statistically significant p-value $\left(r^{2}=0.343, p=0.028\right)$, while the positive correlation between TGF- $\beta$ expression and h-TERT was very weak and statistically insignificant (Table V, Fig. 3).
A

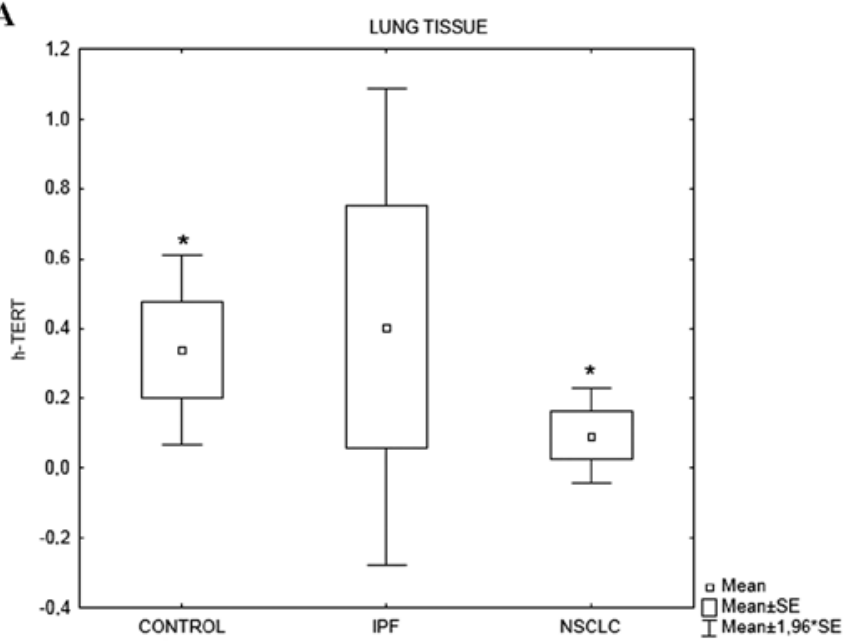

B

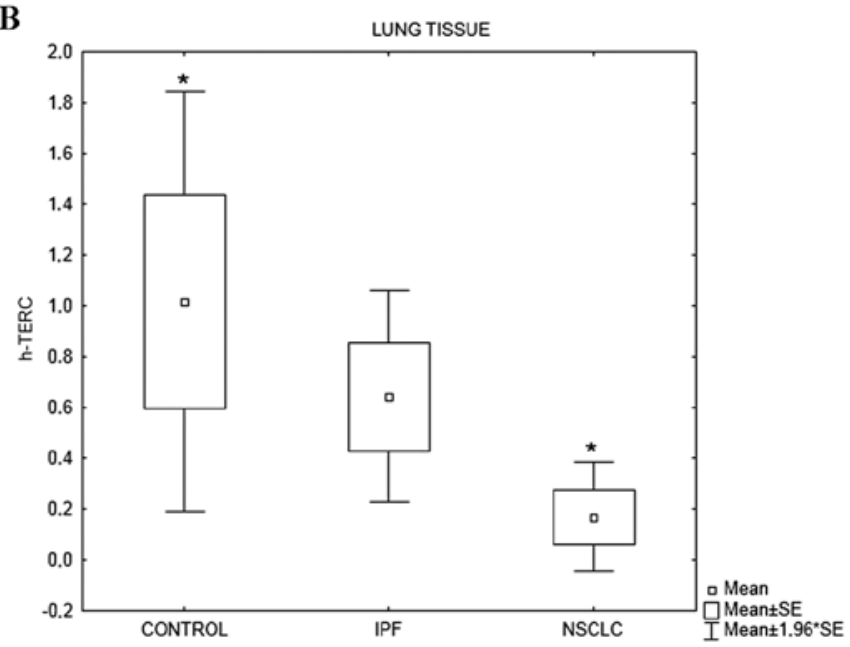

Figure 1. (A) h-TERT and (B) h-TERC relative mRNA expression in the lung tissue samples from the control subjects (parenchyma) and patients with IPF and NSCLC (tumor samples). " p $<0.05$ vs. IPF. NSCLC, non-small cell lung cancer; IFP, idiopathic pulmonary fibrosis.

\section{Discussion}

The main finding of this study is the attenuated expression of both telomerase subunits measured in lung tissue obtained from patients with IPF, compared with lung tissue obtained from patients with NSCLC and control subjects. To our knowledge, this is the first time that h-TERT/h-TERC expression levels have been measured in human lung tissue and BALF.

Fibrogenesis and carcinogenesis are attractive research topics, as although therapeutic efforts have increased over the past decade, few advances have been made (4-6,20). Certain studies have suggested a link between IPF and lung cancer through different pathogenetic mechanisms, such as viral implications, inflammation, coagulation, dysregulated apoptosis, focal hypoxia, activation of oncogenes, genetics and the accumulation of myofibroblasts, as well as extracellular matrix accumulation $(21,22)$. However, diversities in the expression of molecular pathways have also been recently described by ours $(14,23)$ and other study groups (4-6). Previous studies have demonstrated that mutations in telomerase h-TERT and h-TERC 
Table IV. h-TERT and h-TERC relative mRNA expression levels in lung tissue and BALF samples from patients with IPF and NSCLC and the control subjects.

A, Lung tissue samples

\begin{tabular}{lcccc}
\hline & Control $(\mathrm{n}=21)$ & IPF $(\mathrm{n}=29)$ & NSCLC $(\mathrm{n}=10)$ & $\mathrm{p}-\mathrm{value}$ \\
\hline h-TERT & $0.01[0-0.35]$ & $0.00[0-0]$ & $0.695[0-2.34]$ & 0.036 \\
h-TERC & $1.12[0-2.66]$ & $0.00[0-0]$ & $0.97[0.68-2.88]$ & 0.002 \\
TGF- $\beta$ & $0.7390[0.1280-3.220]$ & $194[0.0015-3070]$ & $0.00[0.00-0.03]$ & $<0.0001$ \\
\hline
\end{tabular}

B, BALF samples

\begin{tabular}{lcccc}
\hline & Control $(\mathrm{n}=12)$ & IPF $(\mathrm{n}=23)$ & NSCLC $(\mathrm{n}=31)$ & $\mathrm{p}-\mathrm{v}$ alue \\
\hline h-TERT & $0.00[0-0.02]$ & $0.09[0-0.23]$ & $0.00[0-0.01]$ & 0.012 \\
h-TERC & $0.005[0-0.385]$ & $0.44[0-1.21]$ & $0.06[0-0.73]$ & 0.07 \\
TGF- $\beta$ & $100.3[1.51-533.6]$ & $199.2[1.873-11200000]$ & $0.0020[0-2.7933]$ & $<0.0001$ \\
\hline
\end{tabular}

Data are presented as the median (lower-upper quartiles) and a value of $\mathrm{p}<0.05$ was considered to indicate a statistically significant difference, Kruskal-Wallis test. NSCLC, non-small cell lung cancer; IFP, idiopathic pulmonary fibrosis; BALF, bronchoalveolar lavage fluid.

Table V. Correlation of h-TERT and h-TERC relative mRNA expression with TGF- $\beta$ relative mRNA expression in patients with IPF and NSCLC.

A

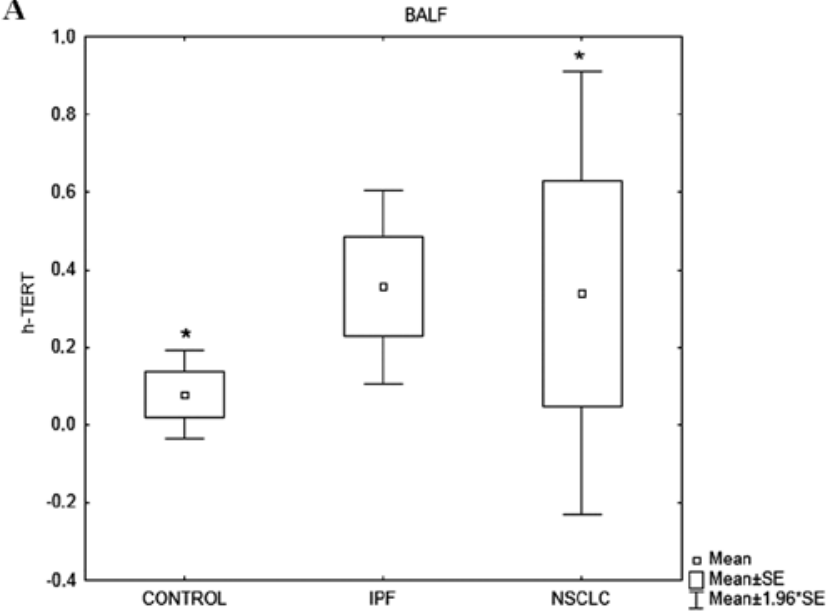

\section{Group}

Telomerase gene per biological sample

\begin{tabular}{ll} 
Group \\
\hline IPF & NSCLC
\end{tabular}

TGF- $\beta$ relative mRNA expression in BALF correlated with h-TERT

TGF- $\beta$ relative mRNA expression in BALF correlated with h-TERC

$\mathrm{r}^{2}=0.015, \mathrm{r}=0.3012, \mathrm{p}=\mathrm{NS} \quad \mathrm{r}^{2}=0.006, \mathrm{r}=0.055, \mathrm{p}=\mathrm{NS}$

作

TGF- $\beta$ relative mRNA expression in lung tissue correlated with h-TERC $r^{2}=0.089, r=-0.065, p=N S \quad r^{2}=0.343, r=0.585, p=0.028$

NSCLC, non-small cell lung cancer; IFP, idiopathic pulmonary fibrosis. BALF, bronchoalveolar lavage fluid.

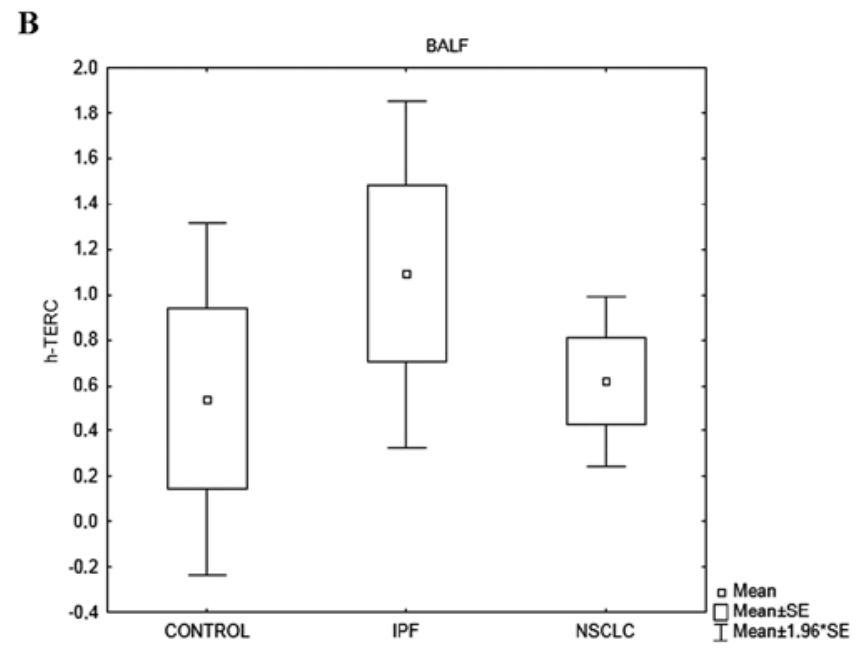

B 


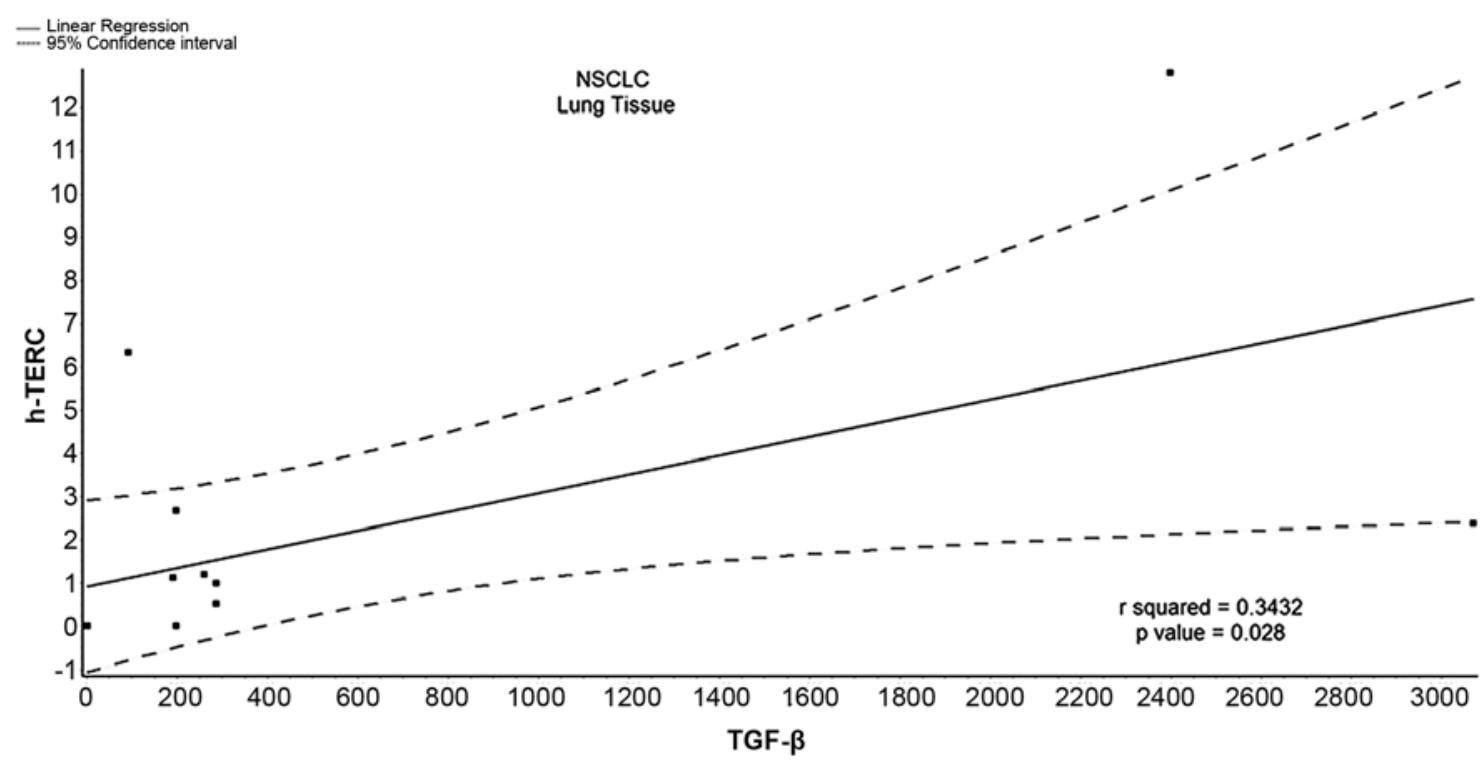

Figure 3. Correlation of h-TERC relative mRNA expression with TGF- $\beta$ relative mRNA expression in lung tissue samples from patinets with NSCLC (tumor samples) ( $\mathrm{r} 2=0.343, \mathrm{p}=0.028)$. NSCLC, non-small cell lung cancer.

genes cause a shortening of telomere lengths, and account for approximately $10 \%$ of familial IPF cases (24-26).

In this study, h-TERT mRNA expression was downregulated in the lung tissue samples obtained from patients with IPF, which is the most novel finding of our study. Our study was not designed to unravel the mechanism of h-TERT downregulation in this disease. Nevertheless, some speculations are worth pursuing. Decreased h-TERT expression levels may represent decreased h-TERT mRNA transcription due to downregulation by a variety of factors, such as p53, TGF- $\beta$, Wilms tumor-1 (Wt-1) and murine double minute 2 (Mdm2) (27), which have been implicated in the pathogenesis of IPF. These factors have been shown to negatively regulate h-TERT, thus limiting telomerase activation (28). However, it should be acknowledged that h-TERT activity may also be regulated by alternative splicing and post-translational modifications (27). As h-TERT is the catalytic subunit of the telomerase complex it can be hypothesized that the attenuated expression also involves the downregulation of telomerase activity. Another possible explanation for the attenuated h-TERT expression measured in our patients compared with the healthy controls is the existence of mutations in the h-TERT gene in patients with IPF, which would inhibit telomerase activity. However, telomerase mutations in sporadic IPF cases are rare, detected in only 1 out of 100 patients in the general population (29).

In contrast to the lung tissue samples, h-TERT expression was augmented in the BALF samples compared with the healthy controls. This suggests that BALF is not representative of telomerase expression in tissue and cannot be recommended as surrogate material of telomerase expression determination in patients with IPF. The reason for the increased expression in BALF is not clear and cannot be addressed by the results of our study. It is highly possible that macrophages and neutrophils which constitute the major cell subpopulations in BALF from patients with IPF (30) exhibit increased h-TERT expression, which is in accordance with previous results from our group, where bone marrow-derived mesenchymal stem cells from patients with IPF showed a trend for increased h-TERT expression compared with the healthy controls (31). h-TERC mRNA expression levels exhibited the same pattern as the h-TERT levels in patients with IPF.

The assessment of $\mathrm{h}$-TERT/h-TERC mRNA expression in lung tissue and BALF from patients with NSCLC revealed a profile similar to that of the control group for both subunits. In the lung tissue samples, a trend for increased h-TERC expression was observed compared with the controls, although statistical significance was not achieved. However, when comparing NSCLC with IPF, significant differences were observed. More explicitly, both h-TERT and h-TERC expression levels were significantly decreased in the tissue samples from patients with IPF compared with the patients with NSCLC, suggesting that telomerase genes play a differential role in fibrogenesis and carcinogenesis. An analysis of the BALF samples revealed increased h-TERT expression levels in patients with IPF compared with patients with NSCLC, again suggesting differences in the telomerase pathway between these two diseases.

This study further explored the correlation between a known fibrogenic gene, namely TGF- $\beta$, and h-TERT, as well as h-TERC gene expression. The results revealed no significant correlation between fibrotic gene expression, represented by TGF- $\beta$, and telomerase gene expression in the IPF samples. Of note, TGF- $\beta$ expression in the lung tissue samples from patients with NSCLC was found to significantly correlate with h-TERC gene expression. Alveolar epithelial cells exposed to TGF- $\beta$ have been shown to gradually lose epithelial markers, such as cytokeratin, and acquire specific mesenchymal markers, such as $\alpha$ smooth muscle actin ( $\alpha$-SMA), vimentin and type I collagen (6). In pulmonary fibrosis, epithelial cells surrounding fibroblast foci express both epithelial and mesenchymal markers, suggesting that epithelial-mesenchymal transition (EMT) occurs in those areas of lung tissue, supporting an active role for EMT in lung fibrogenesis. As EMT is a form of metaplasia, it is also involved in the early steps of carcinogenesis and cancer cell invasion (6). 
Recent data from an in vitro study indicate that hTERT overexpression promotes the EMT of cancer cells, thereby contributing to lung cancer progression through a TGF- $\beta$ - and $\beta$-cateninmediated pathway (32).

Implications and limitations. In this study, we measured $\mathrm{h}$-TERT/h-TERC expression in both BALF and lung tissue samples. Thus, although we did not determine the cells of origin, combining both tissue and lavage fluid allowed us to provide complementary information that elucidates the expression pattern of telomerase in these diseases. Possible cells of origin of the h-TERT/h-TERC expression from our BALF and lung tissue samples are alveolar epithelial cells (30), alveolar macrophages and leukocytes infiltrating within the lung vessels or the interstitium. Our group has previously demonstrted that BALF epithelial cells exhibit genetic instability [microsatellite instability (MSI)/loss of heterozygosity (LOH)] compared with leukocytes/macrophages (33), which suggests that the reduced telomerase expression in epithelial cells may contribute to the attenuated telomerase expression in the lungs of patients with IPF. One further limitation of the present study was that we did not confirm these findings on the protein level in order to exclude the possibility of non-coding RNA at the post-transcriptional level. Further studies are required in order to clarify the cell(s) of origin of telomerase expression in the healthy and diseased human lung. We did not measure telomere length, as we did not study separate cell subpopulations of BALF or lung tissue samples, which is a limitation of our study. Certain data indicate that shorter telomeres in peripheral blood lymphocytes positively correlate with telomere length measured in alveolar epithelial cells from the same individuals (29). Yet, it is not certain that the degree of telomere shortening observed in circulating leukocytes is representative of the telomere length of resident lung cells of the same subjects (34).

In conclusion, in the present study, we demonstrate that both h-TERT and h-TERC mRNA expression is downregulated in lung tissue from patients with IPF compared with healthy controls. The activation of attenuated telomerase genes in IPF has been implicated as a potential therapeutic strategy $(31,35,36)$. Moreover, h-TERT and h-TERC expression levels were found to be significantly decreased in tissue samples from patients with IPF compared with the patients with NSCLC. These results do not provide support for a common pathway hypothesis concerning the telomerase pathway, but rather reveal distinct telomerase activation profiles between NSCLC and IPF. However, further studies are required to evaluate telomere length in both diseases.

\section{References}

1. Raghu G: Idiopathic pulmonary fibrosis: guidelines for diagnosis and clinical management have advanced from consensus-based in 2000 to evidence-based in 2011. Eur Respir J 37: 743-746, 2011.

2. Wuyts WA, Agostini C, Antoniou K, et al: The pathogenesis of pulmonary fibrosis: a moving target. Eur Respir J 41: 1207-1218, 2013.

3. Calado RT and Young NS: Telomere diseases. N Engl J Med 361: 2353-2365, 2009.

4. Vancheri C, Failla M, Crimi N and Raghu G: Idiopathic pulmonary fibrosis: a disease with similarities and links to cancer biology. Eur Respir J 35: 496-504, 2010.
5. Vancheri $\mathrm{C}$ and Du Bois RM: A progression-free end-point for idiopathic pulmonary fibrosis trials: lessons from cancer. Eur Respir J 41: 262-269, 2013.

6. Vancheri C: Idiopathic pulmonary fibrosis: an altered fibroblast proliferation linked to cancer biology. Proc Am Thorac Soc 9: 153-157, 2012.

7. Collins K and Mitchell JR: Telomerase in the human organism. Oncogene 21: 564-579, 2002.

8. Fernandez-Garcia I, Ortiz-de-Solorzano C and Montuenga LM: Telomeres and telomerase in lung cancer. J Thorac Oncol 3: 1085-1088, 2008.

9. Broccoli D, Young JW and De Lange T: Telomerase activity in normal and malignant hematopoietic cells. Proc Natl Acad Sci USA 92: 9082-9086, 1995.

10. Wright WE, Piatyszek MA, Rainey WE, Byrd W and Shay JW: Telomerase activity in human germline and embryonic tissues and cells. Dev Genet 18: 173-179, 1996.

11. Kim NW, Piatyszek MA, Prowse KR, et al: Specific association of human telomerase activity with immortal cells and cancer. Science 266: 2011-2015, 1994.

12. Diaz de Leon A, Cronkhite JT, Katzenstein AL, et al: Telomere lengths, pulmonary fibrosis and telomerase (TERT) mutations. PLoS One 5: e10680, 2010.

13. American Thoracic Society: Idiopathic pulmonary fibrosis: diagnosis and treatment. International consensus statement. American Thoracic Society (ATS) and the European Respiratory Society (ERS). Am J Respir Crit Care Med 161: 646-664, 2000.

14. Margaritopoulos GA, Antoniou KM, Soufla G, Vassalou E, Spandidos DA and Siafakas NM: Yin Yang-1 (YY-1) expression in idiopathic pulmonary fibrosis. J Recept Signal Transduct Res 31: 188-191, 2011

15. Mehrad B, Burdick MD, Zisman DA, et al: Circulating peripheral blood fibrocytes in human fibrotic interstitial lung disease. Biochem Biophys Res Commun 353: 104-108, 2007.

16. Samara KD, Antoniou KM, Karagiannis K, et al: Expression profiles of Toll-like receptors in non-small cell lung cancer and idiopathic pulmonary fibrosis. Int J Oncol 40: 1397-1404, 2012.

17. Antoniou KM, Soufla G, Proklou A, et al: Different activity of the biological axis VEGF-Flt-1 (fms-like tyrosine kinase 1) and CXC chemokines between pulmonary sarcoidosis and idiopathic pulmonary fibrosis: a bronchoalveolar lavage study. Clin Dev Immunol 2009: 537929, 2009.

18. Margaritopoulos GA, Antoniou KM, Karagiannis K, et al: Investigation of Toll-like receptors in the pathogenesis of fibrotic and granulomatous disorders: a bronchoalveolar lavage study. Fibrogenesis Tissue Repair 3: 20, 2010.

19. Antoniou KM, Soufla G,Lymbouridou R, et al: Expression analysis of angiogenic growth factors and biological axis CXCL12/CXCR4 axis in idiopathic pulmonary fibrosis. Connect Tissue Res 51: 71-80, 2010.

20. Margaritopoulos GA, Romagnoli M, Poletti V, Siafakas NM, Wells AU and Antoniou KM: Recent advances in the pathogenesis and clinical evaluation of pulmonary fibrosis. Eur Respir Rev 21: 48-56, 2012.

21. Lasithiotaki I, Antoniou KM, Derdas SP, et al: The presence of merkel cell polyomavirus is associated with deregulated expression of BRAF and Bcl-2 genes in non-small cell lung cancer. Int J Cancer 133: 604-611, 2013.

22. Lasithiotaki I, Antoniou KM, Vlahava VM, et al: Detection of herpes simplex virus type-1 in patients with fibrotic lung diseases. PLoS One 6: e27800, 2011.

23. Antoniou KM, Margaritopoulos GA, Soufla G, et al: Expression analysis of Akt and MAPK signaling pathways in lung tissue of patients with idiopathic pulmonary fibrosis (IPF). J Recept Signal Transduct Res 30: 262-269, 2010.

24. Wang Y, Kuan PJ, Xing C, et al: Genetic defects in surfactant protein A2 are associated with pulmonary fibrosis and lung cancer. Am J Hum Genet 84: 52-59, 2009.

25. Tsakiri KD, Cronkhite JT, Kuan PJ, et al: Adult-onset pulmonary fibrosis caused by mutations in telomerase. Proc Natl Acad Sci USA 104: 7552-7557, 2007.

26. Diaz de Leon A, Cronkhite JT, Yilmaz C, et al: Subclinical lung disease, macrocytosis, and premature graying in kindreds with telomerase (TERT) mutations. Chest 140: 753-763, 2011.

27. Flores I, Benetti R and Blasco MA: Telomerase regulation and stem cell behaviour. Curr Opin Cell Biol 18: 254-260, 2006.

28. Lin SY and Elledge SJ: Multiple tumour suppressor pathways negatively regulate telomerase. Cell 113: 881-889, 2003.

29. Alder JK, Chen JJ, Lancaster L, et al: Short telomeres are a risk factor for idiopathic pulmonary fibrosis. Proc Natl Acad Sci USA 105: 13051-13056, 2008. 
30. Meyer KC, Raghu G, Baughman RP, et al; for the American Thoracic Society Committee on BAL in Interstitial Lung Disease: An official American Thoracic Society clinical practice guideline: the clinical utility of bronchoalveolar lavage cellular analysis in interstitial lung disease. Am J Respir Crit Care Med 185: 1004-1014, 2012.

31. Antoniou KM,Margaritopoulos GA,Proklou A, et al: Investigation of telomerase/telomeres system in bone marrow mesenchymal stem cells derived from IPF and RA-UIP. J Inflamm (Lond) 9: 27, 2012 .

32. Liu Z, Li Q, Li K, et al: Telomerase reverse transcriptase promotes epithelial-mesenchymal transition and stem cell-like traits in cancer cells. Oncogene 32: 4203-4213, 2012.
33. Samara KD, Tzortzaki EG, Neofytou E, et al: Somatic DNA alterations in lung epithelial barrier cells in COPD patients. Pulm Pharmacol Ther 23: 208-214, 2010.

34. Cronkhite JT, Xing C, Raghu G, et al: Telomere shortening in familial and sporadic pulmonary fibrosis. Am J Respir Crit Care Med 178: 729-737, 2008.

35. Antoniou KM, Papadaki HA, Soufla G and Siafakas NM: Short telomeres and treatment of pulmonary fibrosis: implications for early intervention. Am J Respir Crit Care Med 179: 970, 2009.

36. Garcia CK: Idiopathic pulmonary fibrosis: update on genetic discoveries. Proc Am Thorac Soc 8: 158-162, 2011. 